\title{
Defects and Their Removal in Block Copolymer Thin Film Simulations
}

\author{
AUGUST W. BOSSE, ${ }^{1}$ SCOTT W. SIDES, ${ }^{2}$ KIRILL KATSOV, ${ }^{3}$ CARLOS J. GARCÍA-CERVERA, ${ }^{4}$ GLENN H. FREDRICKSON ${ }^{5}$ \\ ${ }^{1}$ Department of Physics, University of California, Santa Barbara (UCSB), California \\ ${ }^{2}$ Tech-X Corporation, Boulder, Colorado \\ ${ }^{3}$ Materials Research Laboratory, UCSB, California \\ ${ }^{4}$ Department of Mathematics, UCSB, California \\ ${ }^{5}$ Departments of Chemical Engineering and Materials, UCSB, California
}

Received 15 January 2006; revised 28 March 2006; accepted 29 March 2006

DOI: 10.1002 / polb.20905

Published online in Wiley InterScience (www.interscience.wiley.com).

\begin{abstract}
In recent years, there has been increased interest in using microphaseseparated block copolymer thin films as submicrometer/suboptical masks in next generation semiconductor and magnetic media fabrication. With the goals of removing metastable defects in block copolymer thin film simulations and potentially examining equilibrium defect populations, we report on two new numerical techniques that can be used in field-theoretic computer simulations: (1) a spectral amplitude filter (SF) that encourages the simulation to relax into high symmetry states (representing zero defect states), and (2) different variants of force-biased, partial saddle point Monte Carlo algorithms that allow for barrier crossing toward lower energy defect-free states. Beyond their use for removing defects, the force-biased Monte Carlo algorithms will be seen to provide a promising tool for studying equilibrium defect populations. () 2006 Wiley Periodicals, Inc. J Polym Sci Part B: Polym Phys 44: 2495-2511, 2006

Keywords: diblock copolymers; mean-field theory; microdomain defects; Monte Carlo; self-consistent field theory; simulations; thin films
\end{abstract}

\section{INTRODUCTION}

Microphase-separated block copolymer thin films have emerged as a promising new vehicle for the creation of submicrometer/suboptical masks in next generation semiconductor and magnetic media. ${ }^{1-4}$ Depending on the specific architecture and composition of the block copolymer, the microphase-separated system can yield a variety of ordered microdomains. Microphase separation

Correspondence to: A. W. Bosse (E-mail: awbosse@umail. ucsb.edu)

Journal of Polymer Science: Part B: Polymer Physics, Vol. 44, 2495-2511 (2006)

○) 2006 Wiley Periodicals, Inc. occurs on the $10-\mathrm{nm}$ scale, yet the resulting microdomains often exhibit defect structures that extend to the micrometer scale.

Not surprisingly, large ordered arrays of nanometer-scale microdomains represent a promising lithographic tool. For example, in the case of magnetic media fabrication (flash memory), one might desire a large, regular array of magnetic dots. A phase-separated block copolymer thin film could act as a lithographic mask for the creation of such a well-ordered magnetic array. However, if block copolymer masking techniques are to evolve into a realistic and commercially viable fabrication scheme, one must have control over, or at least an understanding of, defect structures and the factors 
that control long-range lateral order in copolymer thin films.

The problem of controlling and understanding microdomain ordering in block copolymer thin films has garnered much attention from polymer experimentalists. ${ }^{5-7}$ For example, Segalman et al. have shown that a polystyrene- $b$-(2-vinylpyridine) (PS-PVP) block copolymer thin film (with $f_{\mathrm{PVP}}$ $=0.129$ and $N=670$ ), which consists of a single layer of spherical PVP-domains in a PS matrix, behaves as a 2D crystal-hexatic-liquid system, consistent with the predictions of the KosterlitzThouless-Halperin-Nelson-Young (KTHNY) theory of $2 \mathrm{D}$ melting. ${ }^{5}$ According to this theory, melting of a $2 \mathrm{D}$ crystal of microdomains proceeds through two continuous phase transitions, first to a hexatic phase and then into a liquid. Each phase is characterized by a well defined equilibrium distribution of microdomain defects.

In addition to equilibrium defect populations, it is common to observe nonequilibrium distributions of metastable defects. Qualitatively, it is useful to think of metastable states as corresponding to local minima in a free energy landscape and being separated from each other by substantial (at least a few $k_{\mathrm{B}} T$ ) activation barriers. In experiments, one often handles nonequilibrium defects via thermal annealing. In computer simulations, both particle-based ${ }^{8}$ and field-theoretic, ${ }^{9}$ it is typically very difficult to relax toward a defect-free state or an equilibrium defect population because of the time limitations that are inherent to the methods.

There are various field-based simulation methodologies that are successful at yielding defect-free states, including unit-cell calculations by means of finite differences, pseudo-spectral techniques, or the spectral method of Matsen and Schick. ${ }^{9,10}$ These methods require either seeding the simulation cell with a specific microdomain morphology or imposing a specific symmetry so that only certain symmetry-constrained structures can form. However, if one is primarily interested in equilibrium defect populations, or if one wishes to allow symmetries to set in in an unbiased manner (for example, if the symmetries of the microphases are unknown), then one needs to apply large-cell simulations. In such simulations, periodic boundary conditions are typically imposed, but the size of the computational cell is taken to be much larger than the microdomain period.

In the present paper we address two issues that relate to large-cell field theoretic simulations of block copolymers:
- We develop techniques for achieving defectfree mean-field solutions. This is especially important when conducting simulations in a discovery mode, where the microdomain symmetry is not known in advance. The highly defective metastable states obtained in such simulations often do not possess sufficient long-range order to clearly identify the underlying defect-free periodic ground state.

- We develop simulation techniques that can generate equilibrium defect populations, such as those observed by Segalman et al. ${ }^{5}$ in block copolymer thin films.

Consequently, the purpose of this study is to develop computational methods for removing and evolving microdomain defects in unbiased largecell block copolymer simulations. For the purpose of removing defects in block copolymer simulations, we develop two computational methods: (1) a spectral amplitude filtering (SF) algorithm, and (2) several variants of force-biased Monte Carlo (MC) algorithms. The MC methods also appear to be useful in generating equilibrium defect populations, and so address our second objective.

In Background section we provide some background relevant to the problem of microdomain defects in block copolymer thin films. Model and SCFT section details our polymer model, the meanfield approximation leading to self-consistent field theory (SCFT), and our pseudo-spectral numerical methods, including the semi-implicit Seidel (SIS) technique for relaxing mean-field solutions. In Spectral Amplitude Filtering section we introduce and describe an SF algorithm for the elimination of defects. Partial Saddle Point Monte Carlo section describes a family of force-biased MC algorithms (PSP-MC), based on a partial saddlepoint (PSP) approximation to the underlying field theory model. Results section provides a comparison of the ability of these techniques to eliminate microdomain defects in two-dimensional (2D) diblock copolymer thin films. Finally, Discussion and Conclusions section summarizes our results and provides some concluding remarks.

\section{BACKGROUND}

As mentioned earlier, KTHNY theory predicts equilibrium populations of lattice defects in $2 \mathrm{D}$ systems (here "lattice" refers to the characteristic lattice formed by the phase-separated microdomains). A useful way to identify lattice defects 
is via a Voronoi diagram. ${ }^{5}$ The diagram is constructed from the polyhedra formed by perpendicular bisection of the bonds between all neighboring lattice points. The number of sides of the resulting polyhedra indicates the number of nearest neighbors; thus, the Voronoi diagram allows one to quickly identify the coordination of each lattice point (here we define "lattice point" as the centroid of a microdomain).

For the system of interest, an asymmetric $\mathrm{AB}$ diblock copolymer with composition $f>0.5$ (A block volume fraction) in $2 \mathrm{D}$, the copolymer melt can microphase separate into an ordered hexagonal array of B cylinders, depending on the specific values of $f$ and the Flory parameter $\chi_{\mathrm{AB}} \cdot{ }^{10,11} \mathrm{In}$ $2 \mathrm{D}$, the only nonlamellar microphase is a hexagonally ordered cylindrical phase. For a perfect hexagonal lattice, each $\mathrm{B}$ cylinder will have six nearest neighbors. Therefore, topological lattice defects are characterized by microdomains with less than or more than six nearest neighbors; in other words, Voronoi polygons with other than six sides. It is worth noting that the three phases considered in the KTHNY theory can be distinguished by the number and type of nonhexagonal Voronoi polygons. ${ }^{5}$

The standard field theory model of an incompressible block copolymer melt on which our simulations are based $^{9,10,12}$ applies HubbardStratonovich (HS) fields to decouple the various polymers in the system. The mean-field approximation (SCFT) corresponds to a saddle-point approximation for the functional integrals over these fields. To compute SCFT solutions, i.e. to conduct SCFT simulations, it is necessary to have a numerical procedure for solving a Fokker-Planck equation describing the statistical mechanics of a single copolymer in the HS "self-consistent" fields, and a second numerical algorithm for relaxing the fields to approach a saddle point. For the first purpose, we use a second-order accurate, pseudospectral algorithm based on operator splitting. ${ }^{13,14}$ In relaxing the fields, we use a variation of a continuous steepest descent search. ${ }^{9}$ A fictitious time variable is introduced, and at each moment in time the system is moved along the gradient of the energy functional to approach a saddle point.

Such SCFT simulations ultimately terminate at a stable or metastable saddle point solution, corresponding to a mean-field solution of the block copolymer partition function. The continuous steepest descent scheme lacks a mechanism for moving between metastable states (i.e., barrier crossing between saddle points). Although there has been recent work on improving the speed and stability of the steepest descent search, ${ }^{15}$ barrier crossing and the avoidance of metastable, defect-laden states still present a problem.

One possible method of reducing defect populations is to extend the steepest descent search beyond the mean-field approximation (SCFT) and incorporate thermal fluctuations. Such fluctuations may be sufficient to enable barrier crossing and annealing of defect structures. There are various ways to conduct such "field-theoretic simulations," the two most popular of which are MC and complex Langevin (CL) methods. ${ }^{9,16,17}$ Although these methods successfully extend the theory beyond the mean-field approximation, they have problems of their own. First, and perhaps most important, any given configuration obtained by the stochastic methods are not solutions per se, but are snapshots suitable for calculating statistical averages. Here we are primarily concerned with "ground-state" SCFT solutions. Second, full MC/CL sampling requires a doubling in the number of field variables used when compared with SCFT. Third, the CL approach is subject to accuracy and stability limitations that can make the simulations prohibitively slow for our purposes (although there has been recent progress in this issue ${ }^{18}$ ). Finally, full MC simulations of field-theoretic models of polymers exhibit a "sign problem", 17 that can slow or even prevent convergence of this approach.

With the primary goal of removing metastable defects in SCFT simulations of block copolymer thin films, we examine two algorithms: (1) an SF algorithm that dynamically amplifies symmetries developing in the system and encourages the steepest descent search to move in the appropriate direction, and (2) a family of force-biased MC algorithms, which introduce controlled noise to the steepest descent search, thus potentially facilitating barrier crossing. As for our secondary goal, the $\mathrm{MC}$ algorithms developed here satisfy detailed balance, and so they may prove useful in simulating equilibrium defect populations as predicted by KTHNY theory and observed by Segalman et al. ${ }^{5}$

We note that Knoll et al. have recently reported on the use of dynamic density functional theory to examine the kinetics of the phase transition between parallel cylinders and perforated lamella in block copolymer thin films. ${ }^{19}$ The results are interesting and shed light on how defects move and annihilate under diffusive dynamics during the course of such phase transitions. Nonetheless, the Knoll et al. simulations focused on 
microdomain kinetics during a block copolymer phase transition. Here we are concerned with rapidly locating mean-field solutions through the use of fictitious, nonconservative dynamics, with strategies for defect removal, and with the efficient sampling of equilibrium defect populations.

\section{MODEL AND SCFT}

Both of our microdomain defect removal algorithms are built on top of a standard field theory model for an incompressible $\mathrm{AB}$ diblock copolymer melt. ${ }^{10,17}$ In this section we provide a short review of the basic polymer model, the SCFT (saddle point) approximation, and the SIS algorithm for obtaining saddle point solutions.

\section{Block Copolymer Model}

We consider $n$ monodisperse $\mathrm{AB}$ diblock copolymers in a volume $V$. The index of polymerization is denoted by $N$, and the fraction of A monomer is denoted by $f$. We assume the statistical segment lengths of the two polymers are equal $b_{\mathrm{A}}=b_{\mathrm{B}}=b$; therefore, in three spatial dimensions, the unperturbed radius of gyration is given by $R_{g 0}^{2}=b^{2} N / 6$. We restrict our attention to incompressible polymer melts, and thus the fixed, average monomer density is given by $\rho_{0}=n N / V$. The block copolymers are modeled using the continuous Gaussian chain model with space curves $\mathbf{r}_{\alpha}(s)$. Here $\alpha$ $=1,2, \ldots, n$ is the polymer index, and $s \in[0,1]$ is a polymer contour length variable that runs from $s=0$ at the beginning of the A side to $s=1$ at the end of the $\mathrm{B}$ side. The canonical partition function is given by (here and throughout we set $k_{\mathrm{B}} T=1$ )

$$
Z=\int\left(\prod_{\alpha=1}^{n} \mathcal{D} \mathbf{r}_{\alpha}\right) \delta\left[\hat{\rho}_{\mathrm{A}}+\hat{\rho}_{\mathrm{B}}-\rho_{0}\right] \exp \left(-H_{0}-H_{\mathrm{I}}\right)
$$

where $H_{0}$ is the harmonic chain stretching penalty,

$$
H_{0}=\frac{1}{4 R_{g 0}^{2}} \sum_{\alpha=1}^{n} \int_{0}^{1} d s\left|\frac{d \mathbf{r}_{\alpha}(s)}{d s}\right|^{2},
$$

and $H_{\mathrm{I}}$ is the Flory monomer-monomer interaction,

$$
H_{\mathrm{I}}=\frac{\chi}{\rho_{0}} \int_{V} d \mathbf{r} \hat{\rho}_{\mathrm{A}}(\mathbf{r}) \hat{\rho}_{\mathrm{B}}(\mathbf{r}) .
$$

Here $\chi=\chi_{\mathrm{AB}}$ is the Flory interaction parameter for interactions between $\mathrm{A}$ and $\mathrm{B}$ monomers.
The microscopic A and B monomer densities are defined as follows:

$$
\begin{aligned}
& \hat{\rho}_{\mathrm{A}}(\mathbf{r})=N \sum_{\alpha=1}^{n} \int_{0}^{f} d s \delta\left(\mathbf{r}-\mathbf{r}_{\alpha}(s)\right), \\
& \hat{\rho}_{\mathrm{B}}(\mathbf{r})=N \sum_{\alpha=1}^{n} \int_{f}^{1} d s \delta\left(\mathbf{r}-\mathbf{r}_{\alpha}(s)\right) .
\end{aligned}
$$

In the partition function eq $1, \int\left(\prod_{\alpha=1}^{n} \mathcal{D} \mathbf{r}_{\alpha}\right)$ represents a functional integral over all chain configurations of all of the polymers, and $\delta\left[\hat{\rho}_{\mathrm{A}}+\hat{\rho}_{\mathrm{B}}-\rho_{0}\right]$ is a delta functional that enforces the incompressibility constraint of the melt, $\hat{\rho}_{\mathrm{A}}(\mathbf{r})+\hat{\rho}_{\mathrm{B}}(\mathbf{r})=\rho_{0}$ at all points.

The next step is to perform a HubbardStratonovich transformation on the partition function. This replaces monomer-monomer interactions (contained in $H_{\mathrm{I}}$ ) with a single polymer interacting with an external field. First we introduce a change of variables

$$
\hat{\rho}_{ \pm}(\mathbf{r})=\hat{\rho}_{\mathrm{A}}(\mathbf{r}) \pm \hat{\rho}_{\mathrm{B}}(\mathbf{r}) .
$$

The change of variables allows us to write

$$
H_{\mathrm{I}}=-\frac{1}{4} \frac{\chi}{\rho_{0}} \int_{V} d \mathbf{r}\left[\hat{\rho}_{-}(\mathbf{r})-(2 f-1) \rho_{0}\right]^{2},
$$

up to a constant shift in energy. We can now convert eq 1 into a field theory by performing a Hubbard-Stratonovich transformation

$$
\begin{aligned}
\exp & {\left[-\frac{1}{4 a} \int_{V} d \mathbf{r} b^{2}(\mathbf{r})\right] } \\
& =\int \mathcal{D} w_{-} \exp \left[\int_{V} d \mathbf{r}\left[-a w_{-}^{2}(\mathbf{r})+b(\mathbf{r}) w_{-}(\mathbf{r})\right]\right]
\end{aligned}
$$

and using the exponential representation of the delta functional,

$$
\delta[c]=\int \mathcal{D} w_{+} \exp \left[-i \int d \mathbf{r} w_{+}(\mathbf{r}) c(\mathbf{r})\right]
$$

Here $b(\mathbf{r})=\hat{\rho}_{-}(\mathbf{r})-(2 f-1) \rho_{0}, a=\rho_{0} / \chi$, and $c(\mathbf{r})=\hat{\rho}_{\mathrm{A}}(\mathbf{r})+\hat{\rho}_{\mathrm{B}}(\mathbf{r})-\rho_{0}$. After some simple algebra and rescalings, we obtain

$$
Z=\int \mathcal{D} W_{+} \mathcal{D} W_{-} \exp \left(-H\left[W_{+}, W_{-}\right]\right)
$$


where

$$
\begin{aligned}
& H\left[W_{+}, W_{-}\right]=C \int_{V} d \mathbf{x}\left[-i W_{+}(\mathbf{x})+(2 f-1) W_{-}(\mathbf{x})\right. \\
& \left.\quad+W_{-}^{2}(\mathbf{x}) / \chi N\right]-C V \log \mathcal{Q}\left[i W_{+}-W_{-}, i W_{+}+W_{-}\right] .
\end{aligned}
$$

Here, $\mathbf{x}$ is the dimensionless spatial coordinate $\mathbf{x}=\mathbf{r} / R_{g 0}$ and $C=\left(\rho_{0} / N\right) R_{g 0}^{3}$ is a dimensionless chain concentration. From here on, all lengths are expressed in units of $R_{g 0}$ (e.g., $V / R_{g 0}^{3} \rightarrow V$ ). The parameter $C$ can be viewed as an effective coordination number (the average number of chains that penetrate the coil of a given copolymer) and will be seen to control the strength of fluctuations in the system. The conjugate fields $w_{ \pm}$, defined in eqs 8 and 9 , are also given a new normalization: $W_{ \pm}=N w_{ \pm}$.

In the Hamiltonian eq 11, $\mathcal{Q}$ represents the partition function for a single $\mathrm{AB}$ diblock copolymer in an external field. The A block interacts with the field $W_{\mathrm{A}}=i W_{+}-W_{-}$and the B block interacts with the field $W_{\mathrm{B}}=i W_{+}+W_{-}$. We can calculate $\mathcal{Q}$ using the propagator, $q\left(\mathbf{x}, 1 ;\left[W_{\mathrm{A}}, W_{\mathrm{B}}\right]\right)$ :

$$
\mathcal{Q}\left[W_{\mathrm{A}}, W_{\mathrm{B}}\right]=\frac{1}{V} \int_{V} d \mathbf{x} q\left(\mathbf{x}, 1 ;\left[W_{\mathrm{A}}, W_{\mathrm{B}}\right]\right) .
$$

The propagator $q\left(\mathbf{x}, 1 ;\left[W_{\mathrm{A}}, W_{\mathrm{B}}\right]\right)$ gives the probability density of finding a polymer whose chain end is at position $\mathbf{x}$; it satisfies the following modified diffusion equation:

$$
\frac{\partial}{\partial s} q(\mathbf{x}, s)=\nabla^{2} q(\mathbf{x}, s)-\psi(\mathbf{x}, s) q(\mathbf{x}, s),
$$

subject to the initial condition $q(\mathbf{x}, 0)=1$ and with

$$
\psi(\mathbf{x}, s)=\left\{\begin{array}{l}
i W_{+}(\mathbf{x})-W_{-}(\mathbf{x}), 0<s<f \\
i W_{+}(\mathbf{x})+W_{-}(\mathbf{x}), f<s<1 .
\end{array}\right.
$$

The microscopic monomer densities are computed using the propagator as follows:

$$
\begin{aligned}
& \phi_{\mathrm{A}}\left(\mathbf{x} ;\left[W_{\mathrm{A}}, W_{\mathrm{B}}\right]\right)=\frac{1}{\mathcal{Q}} \int_{0}^{f} d s q(\mathbf{x}, s) q^{\dagger}(\mathbf{x}, 1-s), \\
& \phi_{\mathrm{B}}\left(\mathbf{x} ;\left[W_{\mathrm{A}}, W_{\mathrm{B}}\right]\right)=\frac{1}{\mathcal{Q}} \int_{f}^{1} d s q(\mathbf{x}, s) q^{\dagger}(\mathbf{x}, 1-s) .
\end{aligned}
$$

Here, $\phi_{\mathrm{J}}\left(\mathbf{x} ;\left[W_{\mathrm{A}}, W_{\mathrm{B}}\right]\right)=\rho_{\mathrm{J}}\left(\mathbf{x} ;\left[W_{\mathrm{A}}, W_{\mathrm{B}}\right]\right) / \rho_{0}, \quad \mathrm{~J}$ $=\mathrm{A}, \mathrm{B}$ is the reduced local monomer density (i.e., the local volume fraction of segments of type $\mathrm{J}$ ), and $q^{\dagger}(\mathbf{x}, s)$ is a backwards propagator. The backwards propagator is needed because the copolymer chain is not symmetric; it satisfies the following equation:

$$
\frac{\partial}{\partial s} q^{\dagger}(\mathbf{x}, s)=\nabla^{2} q^{\dagger}(\mathbf{x}, s)-\psi^{\dagger}(\mathbf{x}, s) q^{\dagger}(\mathbf{x}, s)
$$

subject to the initial condition $q^{\dagger}(\mathbf{x}, 0)=1$ and with

$$
\psi^{\dagger}(\mathbf{x}, s)=\left\{\begin{array}{l}
i W_{+}(\mathbf{x})+W_{-}(\mathbf{x}), 0<s<1-f \\
i W_{+}(\mathbf{x})-W_{-}(\mathbf{x}), 1-f<s<1 .
\end{array}\right.
$$

\section{Self-Consistent Field Theory}

Up to this point we have performed a series of exact transformations on the original polymer partition function eq 1 to yield a new, formally equivalent field theory with partition function given by eq 10. We now shift our attention to mean field approximations to the partition function given by eq 10.

The factor $C$ appears in front of all terms in eq 11; therefore, for $C \rightarrow \infty$ we can use the method of steepest descent to validate saddle point solutions of the above theory. The saddle point solutions represent mean-field approximations to eq $10 .{ }^{17}$ The saddle point equations are given by

$$
\left.\frac{\delta H\left[W_{+}, W_{-}\right]}{\delta W_{ \pm}}\right|_{\widetilde{W}_{ \pm}}=0
$$

where $\widetilde{W}_{ \pm}$are the saddle-point configurations of the fields $W_{ \pm}$.

Equation 19 represents four equations, one for each component of the complex fields $\widetilde{W}_{ \pm}$; however, it is known that the saddle-point configuration of $W_{+}$is strictly imaginary and that of $W_{-}$is strictly real. ${ }^{17}$ Therefore, we introduce the real valued pressure field $\Xi=i \widetilde{W}_{+}=-{ }_{\widetilde{W}} \operatorname{Im}\left[\widetilde{W}_{+}\right]$and the real valued exchange field $W=\widetilde{W}_{-}=\operatorname{Re}\left[\widetilde{W}_{-}\right]$.

A continuous steepest descent search is the simplest and one of the most efficient ways to solve the saddle point equations. As mentioned earlier, we introduce a fictitious time variable $t$, and at each time we move the field values in the direction of the gradient of the Hamiltonian. By combining eqs 11 and 19, the saddle point equations are given by

$$
\frac{\delta H[\Xi, W]}{\delta \Xi(\mathbf{x})}=C\left[\phi_{\mathrm{A}}(\mathbf{x})+\phi_{\mathrm{B}}(\mathbf{x})-1\right]=0,
$$


and

$$
\begin{aligned}
\frac{\delta H[\Xi, W]}{\delta W(\mathbf{x})}=C[(2 f-1) & +2 W(\mathbf{x}) / \chi N \\
- & \left.\phi_{\mathrm{A}}(\mathbf{x})+\phi_{\mathrm{B}}(\mathbf{x})\right]=0 .
\end{aligned}
$$

Since the saddle point value $\widetilde{W}_{+}=-i \Xi$ is purely imaginary, the saddle point search is actually a "steepest ascent" in $\Xi$. We write,

$$
\begin{aligned}
\frac{\partial}{\partial t} \Xi(\mathbf{x}, t) & =\frac{\delta H[\Xi, W]}{\delta \Xi(\mathbf{x}, t)}, \\
\frac{\partial}{\partial t} W(\mathbf{x}, t) & =-\frac{\delta H[\Xi, W]}{\delta W(\mathbf{x}, t)} .
\end{aligned}
$$

Clearly, when eqs 22 and 23 are stationary, the saddle point equations are satisfied.

This completes the framework for SCFT. We are able to search for mean-field solutions of the polymer theory by iterating the following steps:

1. Initialize $\Xi(\mathbf{x}, 0)$ and $W(\mathbf{x}, 0)$.

2. Solve the modified diffusion equations: eqs 13 and 17.

3. Calculate $\mathcal{Q}, \phi_{\mathrm{A}}$, and $\phi_{\mathrm{A}}$ using eqs 12,15 , and 16.

4. Update $\Xi(\mathbf{x}, t)$ and $W(\mathbf{x}, t)$ by integrating eqs 22 and 23 forward over a time interval $\Delta t$.

5. Repeat Steps 2-5 until a convergence criterion has been met.

\section{Numerical Methods}

In the aforementioned Step 2, we solve the modified diffusion equations using the pseudospectral operator splitting method developed by Rasmussen and Kalosakas. ${ }^{9,13}$ This is a stable, fast, $\mathcal{O}\left(\Delta s^{2}\right)$ accurate algorithm for solving the modified diffusion equation. In eq 13 we identify the linear operator $\mathcal{L}=\nabla^{2}-\psi(\mathbf{x}, s)$. Formally, we can find the solution at a set of discrete contour locations $s$ by propagating along the polymer chain,

$$
q(\mathbf{x}, s+\Delta s)=e^{\Delta s \mathcal{L}} q(\mathbf{x}, s),
$$

starting from the initial condition $q(\mathbf{x}, 0)=1$.

Motivated by the Baker-Campbell-Hausdorff identity, ${ }^{20}$ we perform an $\mathcal{O}\left(\Delta s^{2}\right)$ splitting of $e^{\Delta s \mathcal{L}}$ :

$$
e^{\Delta s \mathcal{L}}=e^{-\Delta s \psi(\mathbf{x}, s) / 2} e^{\Delta s \nabla^{2}} e^{-\Delta s \psi(\mathbf{x}, s) / 2}+\mathcal{O}\left(\Delta s^{3}\right) .
$$

The potential $\psi(\mathbf{r}, s)$ is diagonal in real space and the Laplacian is diagonal in Fourier space; thus, explicit multiplication by $e^{-\Delta s \psi(\mathbf{x}, s) / 2}$ is evaluated in real space, and the action of $e^{\Delta s \nabla^{2}}$ is evaluated as an explicit multiplication in Fourier space. We move between real and Fourier space using the fast Fourier transform (FFT). ${ }^{21}$

In executing Step 4 of the SCFT algorithm mentioned earlier, our primary goal is to quickly solve the saddle point equations in as stable a manner as possible. The accuracy of the time integration is not important because we seek only stationary states. Furthermore, we wish to avoid being trapped in metastable states that contain defects. In other words, we wish to find the lowest energy, or "ground state" mean-field solution.

It is convenient to discretize the system spatially on a $2 \mathrm{D}$ square lattice with periodic boundary conditions, and we assume that the system is uniform and finite in the third direction. That is, we set

$$
\begin{array}{ll}
x_{i}=i \Delta x, & i=0, \ldots, n_{x}-1, \\
y_{j}=j \Delta y, & j=0, \ldots, n_{y}-1,
\end{array}
$$

where $n_{x}$ and $n_{y}$ are the number of lattice points in the $x$ and $y$ directions respectively. In order to simplify notation, we subsequently use a single, bold face index $\mathbf{i}$ to represent the ordered pair $(i, j)$. The lattice parameters are defined as follows: $\Delta x=L_{x} / n_{x}$ and $\Delta y=L_{y} / n_{y}$, where $L_{x}$ and $L_{y}$ are the dimensions of the system in units of $R_{g 0}$. The dimension of the system in the uniform $z$-direction is denoted by $L_{z}$. Accordingly, the volume of the system is $V=L_{x} L_{y} L_{z}$, the total number of lattice points is $M=n_{x} n_{y}$, and the area per grid point is $\Delta^{2} \mathbf{x}=\Delta x \Delta y$. This particular discretization represents a uniform collocation grid, which allows us to rapidly transform between real and Fourier space via the FFT, as mentioned earlier.

In the $z$-direction we assume that the system is uniform, which roughly corresponds to the thin film morphology that is of interest here. However, under this assumption of uniformity, the film thickness $L_{z}$ always appears in combination with $C$, the dimensionless polymer concentration, and can simply be adsorbed into its definition.

Finally, we also discretize the chain contour variable $s$ :

$$
s_{l}=l \Delta s, \quad l=0, \ldots, n_{s}
$$

where $n_{s}$ is the number of steps along the polymer backbone, and $\Delta s=1 / n_{s}$ is the contour step size. The fictitious time variable $t$ is also sampled 
at discrete intervals:

$$
t_{m}=m \Delta t, \quad m=0, \ldots, n_{t} .
$$

The value of $\Delta t$ chosen depends on the method used to solve the relaxation equations, and $n_{t}$ defines the number of iterations used to relax the SCFT equations.

\section{Semi-implicit Seidel}

Until recently, only simple forward Euler algorithms and related explicit schemes had been employed for solving the relaxation equations, eqs 22 and 23:

$$
\begin{aligned}
\Xi_{\mathbf{i}}^{m+1} & =\Xi_{\mathbf{i}}^{m}+\Delta t \frac{\partial H\left[\Xi^{m}, W^{m}\right]}{\partial \Xi_{\mathbf{i}}^{m}}, \\
W_{\mathbf{i}}^{m+1} & =W_{\mathbf{i}}^{m}-\Delta t \frac{\partial H\left[\Xi^{m}, W^{m}\right]}{\partial W_{\mathbf{i}}^{m}},
\end{aligned}
$$

where superscripts $m$ represent discrete steps in the time variable $t_{m}$. Although the forward Euler algorithm has been successfully applied, it has a restrictive stability threshold; thus, one is often limited to rather small time steps $\Delta t$. It is not uncommon that forward Euler stepping can require many tens-of-thousands of field iterations to obtain a reasonably accurate saddle point solution.

A more stable algorithm for solving the relaxation equations has been recently proposed by Ceniceros and Fredrickson. ${ }^{15}$ There are various ways to interpret this time stepping algorithm (see Refs. 9 and 15 for detailed discussions), but it is implemented by simply adding and subtracting linearized expressions (in $\Xi$ or $W$ ) for the derivatives of the Hamiltonian to the right hand side of eqs 29 and 30 . The subtracted term is evaluated at the current time $m$, and the added term is evaluated at the future time $m+1$. The result is an SIS update scheme that is implemented pseudospectrally in both real and Fourier space. For the present purposes, it is important to realize that the SIS algorithm is significantly more stable than the forward Euler algorithm. As a result, a much larger time step can be used. It is not uncommon for the SIS algorithm to outperform the forward Euler algorithm by one or two orders of magnitude in the number $n_{t}$ of time steps required to achieve an SCFT solution of prescribed accuracy.

Journal of Polymer Science: Part B: Polymer Physics DOI $10.1002 /$ polb

\section{SPECTRAL AMPLITUDE FILTERING}

Although the SIS relaxation method is well suited for quickly finding a stationary saddle point solution, it does not allow barrier crossing. As a result, it is rather susceptible to getting caught in metastable mean-field solutions. In the Results section we provide specific examples of metastable configurations that were reached via an SIS relaxation.

The SF algorithm is designed to be used in conjunction with another relaxation method. The $\mathrm{SF}$ algorithm is relatively intuitive. Consider a relaxed metastable solution of the saddle point equations for a symmetric diblock copolymer with $\chi N$ above the order-disorder transition (ODT). The density profile of the lowest-energy solution for such a system is a lamellar phase with perfect one-dimensional compositional order. However, metastable solutions typically exhibit morphologies that are locally lamellar, but whose long-range order is destroyed by defects such as dislocations and kink grain boundaries. Even with these defects, the Fourier transform of such composition patterns retains some of the symmetry of the lowest energy ordered state. In Fourier space, the defects produce a spreading of the dominant peaks and a roughened landscape in which these peaks are embedded. With this in mind, if we are able to sharpen the large amplitude peaks of the power spectrum and remove the lower amplitude Fourier components, the resulting real space densities will exhibit increased long-range order. A simple "spectral amplitude filter" that zeroes out all Fourier coefficients whose amplitude is less than some fraction of the largest peak amplitude should be effective in this regard.

Figure 1 (left) represents the modulus of the discrete Fourier transform of $W$ (exchange potential), $\left|\widehat{W}_{\mathbf{k}}\right|$, corresponding to a perfect hexagonal array of microdomains (i.e., a composition profile exhibiting no microdomain defects) for a $f=0.7$ and $\chi N=17 \mathrm{AB}$ diblock melt. This defect-free state was reached by applying the spectral amplitude filter described later. Note that the Fourier amplitude spectrum consists of an array of sharp peaks. The location of these peaks corresponds to the hexagonal symmetry of the ordered microdomains: the six largest peaks are equally spaced around and equidistant from the $(0,0)$ mode.

Figure 1 (right) represents the Fourier amplitude spectrum of $W$ corresponding to a disordered array of microdomains (i.e., a composition profile exhibiting multiple microdomain defects) for 

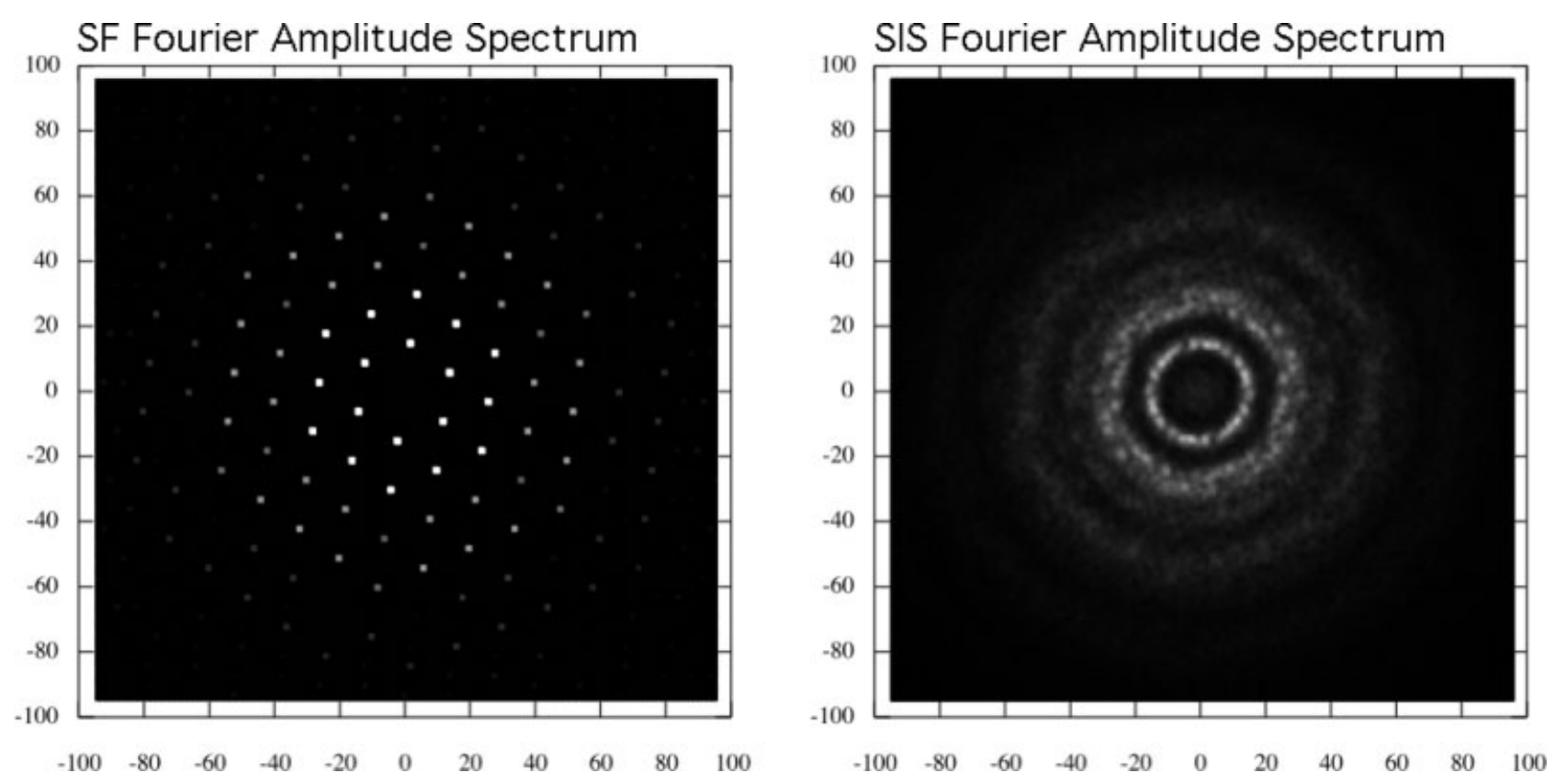

Figure 1. (left) $\left|\widehat{W}_{\mathbf{k}}\right|$ for a defect-free ordered hexagonal lattice of microdomains. This structure was obtained using the SF algorithm combined with SIS relaxation. (right) $\left|\widehat{W}_{\mathbf{k}}\right|$ for a defective array of microdomains. Although the array of microdomains lacks long-range order, short-ranged six-fold symmetry is apparent in the Fourier amplitude spectrum. Although hard to identify, there are six first-order peaks in $\left|\widehat{W}_{\mathbf{k}}\right|$. In both figures, the $x$ and $y$ axes represent discrete modes in Fourier space.

a $f=0.7$ and $\chi N=17 \mathrm{AB}$ diblock melt. Note that although there are still six first-order peaks in the Fourier amplitude spectrum corresponding to hexagonal symmetry, the peaks are dramatically spread out. The SF algorithm is used to isolate and sharpen the largest peaks, and thus produce a Fourier amplitude spectra resembling Figure 1 (left).

To implement the SF algorithm, we identify the highest amplitude peaks in the amplitude spectrum of the $\Xi$ and $W$ fields, then we zero out all wavenumbers that have an amplitude that is less than some fraction $f_{\max }$ of the maximum amplitude. The SF algorithm is summarized below:

1. $\widehat{\Xi}_{\mathbf{k}}=\operatorname{FFT}\left[\Xi_{\mathbf{i}}\right]$ and $\widehat{W}_{\mathbf{k}}=\operatorname{FFT}\left[W_{\mathbf{i}}\right]$.

2. Filter $\widehat{\Xi}_{\mathbf{k}}$ : For all $\mathbf{k}$, if $\left(\left|\widehat{\Xi}_{\mathbf{k}}\right|<f_{\max } \max [|\widehat{\Xi}|]\right)$, then $\widehat{\Xi}_{\mathrm{k}}=0$.

3. Filter $\widehat{W}_{\mathbf{k}}$ : For all $\mathbf{k}$, if $\left(\left|\widehat{W}_{\mathbf{k}}\right|<f_{\max } \max [|\widehat{W}|]\right)$, then $\widehat{W}_{\mathbf{k}}=0$.

4. $\Xi_{\mathbf{i}}=\mathrm{FFT}^{-1}\left[\widehat{\Xi}_{\mathbf{k}}\right]$ and $W_{\mathbf{i}}=\mathrm{FFT}^{-1}\left[\widehat{W}_{\mathbf{k}}\right]$.

This filtering algorithm is applied by alternating with a series of field relaxation steps, so that the system is allowed to relax toward a saddle point, is then filtered, rerelaxed, filtered, etc. Although in principle any suitable relaxation scheme can be paired with the SF, we have found that the SIS algorithm performs particularly well in this role. The number of relaxation steps between applications of the filter and the amplitude threshold $f_{\max }$ of the filter can be tuned to achieve optimal performance of the scheme.

As we shall see, the SF combined with SIS relaxation is a very powerful tool for elimination of defects from 2D hexagonally ordered block copolymer mesophases. However, if the Fourier space representation of $\Xi$ and/or $W$ is particularly feature rich, then the SF can be too aggressive. This has been observed in nontrivial 3D microphases such as the Ia $\overline{3} \mathrm{~d}$ cubic gyroid phase. The gyroid phase has a particularly complicated Fourier amplitude spectrum, with multiple peaks of various amplitudes, each playing a defining role in the gyroid symmetry. When the SF is applied with too tight a threshold $f_{\max }$ in such cases, it is not uncommon to see the filter direct the system into a nongyroid, metastable microphase (e.g., the lamellar phase). ${ }^{22}$ Thus, one must exercise caution when applying this technique to complex block copolymer phases.

Journal of Polymer Science: Part B: Polymer Physics DOI 10.1002/polb 
The SF algorithm is easily extended to more complicated polymer systems; however, its usefulness will depend largely on the complexity of the Fourier amplitude spectrum. One can imagine extending the SF to an $M$-block copolymer system (e.g., $M=3$ corresponding to an $\mathrm{ABC}$ copolymer). In such a system, one would apply the spectral filter to each conjugate potential field $W_{i}$, $\forall i=1,2, \ldots, M$, and to the pressure field $W_{+}$. For $M>2$, we expect a large number of candidate mesophases. As mentioned earlier, however, the spectral filter does not perform well when applied to complex block copolymer phases whose Fourier spectrum contains many secondary peaks of varying intensity that are important to establish the symmetry of the phase. Further study is necessary under such circumstances in order to explore the usefulness of the SF.

\section{PARTIAL SADDLE POINT MONTE CARLO}

In this section we detail another algorithm designed to remove microdomain defects from block copolymer simulations, but that also has the potential for achieving equilibrium defect populations. As outlined earlier, our model of an $\mathrm{AB}$ block copolymer melt includes two potential fields, $W_{+}$and $W_{-}$. The pressure field $W_{+}$effectively enforces the local incompressibility constraint $\phi_{A}(\mathbf{r})+\phi_{B}(\mathbf{r})=1$, and the exchange field $W_{-}$ couples to the A and B composition of the system.

Microdomain defect structures represent local changes in the melt composition. Accordingly, it seems reasonable that by introducing controlled fluctuations to the exchange field relaxation, the system may relax to lower-energy, defect-free configurations. The PSP approximation allows one to add fluctuations in $W_{-}$while instantaneously relaxing $W_{+}$to its mean-field value at the fixed new $W_{-}$configuration. In the following, we define a family of MC methods to introduce fluctuations in $W_{-}$, hence the name partial saddle point Monte Carlo (PSP-MC).

The PSP approximation has been successfully implemented as an approximate way of sampling the equilibrium properties of various fluctuating polymer systems ${ }^{23,24}$ beyond the meanfield approximation. Although not exact, the PSP approximation renders the effective Hamiltonian strictly real and thus side-steps the sign problem present in the full field theory. The PSP approximation is reasonably accurate for the incompressible $\mathrm{AB}$ diblock melt model at large to moderate values of $C,{ }^{23}$ but its general validity in other contexts is questionable. ${ }^{9}$ Here, we simply use the PSP-MC method to add targeted, controlled noise into the system with the hope that this will facilitate defect motion and annihilation. To the extent that the PSP approximation is valid, the scheme potentially also provides a route to simulating and studying equilibrium populations of defects.

The implementation of PSP-MC is straightforward. The functional integrals associated with the pressure field are evaluated using the saddlepoint approximation, while the functional integrals associated with the exchange field are left to be evaluated by MC sampling. A PSP-MC algorithm consists of the following steps, beginning at time $t_{m}$ and field configuration $W^{m}$ :

1. Apply an MC trial move of choice to advance $W^{m}$ to obtain a trial configuration $W^{\mathrm{T}}$.

2. Use the SIS relaxation method to relax $\Xi^{m}$ to its local saddle point value $\Xi^{\mathrm{T}}$ consistent with the trial configuration $W^{\mathrm{T}}$.

3. Using the trial configurations $W^{\mathrm{T}}$ and $\Xi^{\mathrm{T}}$, and with a probability weight that satisfies detailed balance, determine whether to accept the combined trial move $W^{m} \rightarrow W^{\mathrm{T}}$ and $\Xi^{m} \rightarrow \Xi^{\mathrm{T}}$. If the move is accepted, set $W^{m+1}=W^{\mathrm{T}}$ and $\Xi^{m+1}=\Xi^{\mathrm{T}}$; otherwise, set $W^{m+1}=W^{m}$ and $\Xi^{m+1}=\Xi^{m}$.

We note that, in this PSP-MC scheme, trial moves involve global, rather than single site, changes in the field configuration. Single site trial moves in field-theoretic polymer simulations are grossly inefficient because the modified diffusion equations must be re-solved to update the energy, regardless of the number of grid points on which $W^{\mathrm{T}}$ differs from $W^{m}$.

As seen earlier, restricting the pressure field $W_{+}$ to its saddle-point configuration $\Xi=i \widetilde{W}_{+}$yielded a real-valued effective Hamiltonian. Therefore we were able to implement traditional MC algorithms requiring a positive definite probability weight $P \propto e^{-H}$. This idea can be extended to more complicated polymer systems.

In general, one will need to restrict all pressurelike potential fields to their saddle-point configurations in order to ensure a real-valued Hamiltonian. Here, pressure-like refers to potential fields whose saddle-point configuration is purely imaginary. Potential fields with a real-valued saddlepoint configuration are called exchange-like.

We can further examine the requirements of a generalized PSP-MC algorithm by introducing a 
monomer-monomer interaction matrix. Consider a polymer system with $M$ different monomer types (e.g., a $M$-block copolymer), each with a different monomer-monomer interaction parameter $\chi_{i j}$, for $i, j=1,2, \ldots, M$. For such a system, the generalized monomer-monomer interaction energy can be given by

$$
H_{\mathrm{I}}=\frac{1}{2 \rho_{0}} \int_{V} d \mathbf{r} \hat{\rho}^{\mathrm{T}} U \hat{\rho},
$$

where $\hat{\rho}$ is an $M$-dimensional microscopic monomer density vector with components $\hat{\rho}_{i}(\mathbf{r})$, for $i$ $=1,2, \ldots, M$ (each defined by an expression analogous to eqs 4 or 5 ), $\hat{\rho}^{\mathrm{T}}$ is the transpose of $\hat{\rho}$, and $U$ is the $M \times M$ monomer-monomer interaction matrix with components

$$
U_{i j}=\zeta+\chi_{i j}
$$

This matrix captures compressibility with $\zeta$ and the Flory monomer-monomer interactions with $\chi_{i j}$. The $\zeta \rightarrow \infty$ limit yields strict melt incompressibility. The Flory parameter is symmetric, $\chi_{i j}=\chi_{j i}$, and has zero-valued diagonal elements $\chi_{i i}=0$, $\forall i=1,2, \ldots, M$.

The matrix $U$ is symmetric, $U_{i j}=U_{j i}$, and thus possesses real-valued eigenvalues, $\lambda_{j} \in \mathbb{R}$,

$$
U u_{j}=\lambda_{j} u_{j}, \quad \forall i=1,2, \ldots, M
$$

where $u_{j}$ are the eigenvectors of $U$. The sign of the eigenvalues determines whether the corresponding conjugate potential fields $\omega_{j}$ have real-valued or purely imaginary saddle-point configurations.* Specifically, $\omega_{j}$ fields with $\lambda_{j}>0$ are pressure-like, while $\omega_{j}$ fields with $\lambda_{j}<0$ are exchange-like. Once we have identified and classified the potential fields, we can implement a generalized PSP-MC routine: the functional integrals associated with the pressure-like fields are evaluated using the saddle-point approximation, while the functional integrals associated with the exchange-like fields are left to be evaluated by MC sampling.

At present, we have not examined the usefulness of PSP-MC algorithms in the context of more complicated polymer systems. In this paper, we restrict our attention to $\mathrm{AB}$ diblock copolymer melts in $2 \mathrm{D}$.

\footnotetext{
*The eigenvectors are used to define a new density vector $\hat{\varrho}$ with components $\hat{\varrho}_{j}(\mathbf{r})=\left\langle\hat{\rho}(\mathbf{r}), u_{j}\right\rangle$ (analogous to the $\hat{\rho}_{ \pm}$variables from Model and SCFT section). Here $\langle\cdot, \cdot\rangle$ is the inner product. We then perform a Hubbard-Stratonovich transformation, which yields the corresponding conjugate potentials $\omega_{j}$.
}

In this work, we apply two MC methods in the PSP approximation: the force-biased "smart Monte Carlo" (SMC) method of Rossky, Doll, and Friedman, ${ }^{25}$ and a colored-noise (i.e., Fourieraccelerated) variation of the smart MC method. The primary advantage of force-biased MC method is decreased equilibration time in comparison with conventional Metropolis MC. Each force-biased MC kick involves a move along the gradient of the Hamiltonian (in the same way that the steepest descent relaxations move in the direction of the gradient), followed by a random kick. In various tests, we have observed that force-biased MC methods produce a 1-2 order-of-magnitude reduction in equilibration time for field-based simulations, when compared with that by Metropolis MC. Here we detail two variations of the forcebiased smart MC routine: a white noise and a colored noise version.

In the colored noise implementation, we choose a Fourier space noise distribution that has wavenumbers peaked around $q^{*}$, the peak in the diblock structure factor. ${ }^{12}$ This wavenumber roughly corresponds to the fundamental diblock length scale $R_{g 0}$. By carefully selecting the distribution of the noise, it is our hope to not only decrease equilibration time but also allow barrier crossings that correspond to large scale rearrangements of the microdomains toward less defective configurations.

Beginning at an arbitrary time $t_{m}$ and field configurations $\Xi^{m}$ and $W^{m}$, the PSP SMC update procedure is implemented as follows (for more explicit details, particularly with respect to detailed balance and the trial-move acceptance probabilities, see Ref. 25):

1. Evaluate the Hamiltonian $H^{m}=H\left[\Xi^{m}, W^{m}\right]$ and the "W-force" $F_{\mathbf{i}}^{m}=-\partial H^{m} / \partial W_{\mathbf{i}}^{m}$.

2. Generate a trial configuration $W^{\mathrm{T}}$ :

$$
W_{\mathbf{i}}^{\mathrm{T}}=W_{\mathbf{i}}^{m}+\Delta F_{\mathbf{i}}^{m}+R_{\mathbf{i}},
$$

where the random $M$-vector $R_{\mathrm{i}}$ is selected from the distribution

$$
\mathcal{P}\left(\left\{R_{\mathbf{i}}\right\}\right)=\frac{1}{(4 \pi \Delta)^{M / 2}} \exp \left[-\frac{1}{2} \sum_{\mathbf{i}}^{M} \frac{R_{\mathbf{i}}^{2}}{2 \Delta}\right]
$$

3. Relax $\Xi$ to its instantaneous, $W^{\mathrm{T}}$ dependent, saddle-point trial configuration, $\Xi^{\mathrm{T}}$, using SIS updates until the relative error in $H\left[\Xi^{\mathrm{T}}, W^{\mathrm{T}}\right]$ is below a specific, predetermined value $\epsilon$. Note that the force required 
to implement SIS relaxation for the pressure field $\Xi$ is calculated at a fixed value of the exchange field $W=W^{\mathrm{T}}$.

4. Evaluate the Hamiltonian $H^{\mathrm{T}}=H\left[\Xi^{\mathrm{T}}, W^{\mathrm{T}}\right]$, the "W-force" $F_{\mathbf{i}}^{\mathrm{T}}=-\partial H^{\mathrm{T}} / \partial W_{\mathbf{i}}^{\mathrm{T}}$, and the expression

$$
\tau=\frac{\mathcal{P}\left(\left\{W_{\mathbf{i}}^{m}-W_{\mathbf{i}}^{\mathrm{T}}-\Delta F_{\mathbf{i}}^{\mathrm{T}}\right\}\right)}{\mathcal{P}\left(\left\{W_{\mathbf{i}}^{\mathrm{T}}-W_{\mathbf{i}}^{m}-\Delta F_{\mathbf{i}}^{m}\right\}\right)} e^{-\left(H^{\mathrm{T}}-H^{m}\right)} .
$$

5. Select a random number $r \in[0,1]$. If $r \leq \tau$, then accept the trial move and set $\Xi^{m+1}$ $=\Xi^{\mathrm{T}}$ and $W^{m+1}=W^{\mathrm{T}}$, else reject the trial move and set $\Xi^{m+1}=\Xi^{m}$ and $W^{m+1}=W^{m}$.

There are a few technical points worth mentioning. First, the dimensionless polymer concentration (coordination number) $C$ multiplies every term in the Hamiltonian and, thus, controls the extent to which fluctuation corrections to the saddle-point approximation (i.e., SCFT) are important. Simply stated, for large $C$, fluctuations are less important, and the MC procedure will produce results that resemble those of an SCFT simulation. For smaller $C$, fluctuations are more important, and we can expect them to have an appreciable effect on defect populations and defect mobility in the system. Second, the parameter $\Delta$ sets the magnitude of the force and (by detailed balance) the magnitude of the random kick. For optimal performance, $\Delta$ is adjusted to ensure the trial-move acceptance ratio is $\approx 0.5$.

The colored-noise variation of the SMC algorithm represents only a small change to the above-mentioned algorithm. Beginning at an arbitrary time $t_{m}$ and field configurations $\Xi^{m}$ and $W^{m}$, the PSP colored-noise SMC update procedure is implemented as follows:

1. Evaluate the Hamiltonian $H^{m}=H\left[\Xi^{m}, W^{m}\right]$ and the "W-force" $F_{\mathbf{i}}^{m}=-\partial H^{m} / \partial W_{\mathbf{i}}^{m}$.

2. Generate a trial configuration $W^{\mathrm{T}}$ :

$$
W_{\mathbf{i}}^{\mathrm{T}}=W_{\mathbf{i}}^{m}+\sum_{\mathbf{i}^{\prime}}^{M} \Delta_{\left|\mathbf{i}-\mathbf{i}^{\prime}\right|} F_{\mathbf{i}^{\prime}}^{m}+R_{\mathbf{i}}
$$

where the random $M$-vector $R_{\mathbf{i}}$ is selected from the distribution

$$
\begin{aligned}
& \mathcal{P}_{\mathrm{CN}}\left(\left\{R_{\mathbf{i}}\right\}\right) \\
& \propto \exp \left[-\frac{1}{2} \sum_{\mathbf{i}}^{M} \sum_{\mathbf{i}^{\prime}}^{M} R_{\mathbf{i}} R_{\mathbf{i}^{\prime}}\left(\Delta^{-1}\right)_{\left|\mathbf{i}-\mathbf{i}^{\prime}\right|} / 2\right] .
\end{aligned}
$$

3. Relax $\Xi$ to its instantaneous, $W^{\mathrm{T}}$ dependent, saddle-point trial configuration, $\Xi^{\mathrm{T}}$, using the SIS scheme. Continue relaxing until the relative error in $H\left[\Xi^{\mathrm{T}}, W^{\mathrm{T}}\right]$ is below a specific, predetermined value $\epsilon$. Note that the required " $\Xi$-force" is evaluated at fixed exchange potential $W=W^{\mathrm{T}}$.

4. Evaluate the Hamiltonian $H^{\mathrm{T}}=H\left[\Xi^{\mathrm{T}}, W^{\mathrm{T}}\right]$, the "W-force" $F_{\mathbf{i}}^{\mathrm{T}}=-\partial H^{\mathrm{T}} / \partial W_{\mathbf{i}}^{\mathrm{T}}$, and the expression:

$$
\begin{array}{r}
\tau=\frac{\mathcal{P}_{\mathrm{CN}}\left(\left\{W_{\mathbf{i}}^{m}-W_{\mathbf{i}}^{\mathrm{T}}-\sum_{\mathbf{i}^{\prime}}^{M} \Delta_{\left|\mathbf{i}-\mathbf{i}^{\prime}\right|} F_{\mathbf{i}^{\prime}}^{\mathrm{T}}\right\}\right)}{\mathcal{P}_{\mathrm{CN}}\left(\left\{W_{\mathbf{i}}^{\mathrm{T}}-W_{\mathbf{i}}^{m}-\sum_{\mathbf{i}^{\prime}}^{M} \Delta_{\left|\mathbf{i}-\mathbf{i}^{\prime}\right|} F_{\mathbf{i}^{\prime}}^{m}\right\}\right)} \\
\times e^{-\left(H^{\mathrm{T}}-H^{m}\right)} .
\end{array}
$$

5. Select a random number $r \in[0,1]$. If $r \leq \tau$, then accept the trial move and set $\Xi^{m+1}$ $=\Xi^{\mathrm{T}}$ and $W^{m+1}=W^{\mathrm{T}}$, else reject the trial move and set $\Xi^{m+1}=\Xi^{m}$ and $W^{m+1}=W^{m}$.

Again, there are a few technical points worth mentioning. The $M \times M$ matrix $\Delta_{\left|\mathbf{i}-\mathbf{i}^{\prime}\right|}$, which defines the distribution of (spatially) colored noise, is assumed to be translationally invariant and symmetric. Because the discrete Fourier transform of such a matrix is diagonal, the procedure for generating random numbers $R_{\mathrm{i}}$ and evaluating the trial configuration $W^{\mathrm{T}}$ is greatly simplified. Thus, it is natural to perform colored-noise SMC updates in Fourier space. For a function $f_{\mathrm{j}}$ defined on the simulation lattice, we define the Fourier transform

$$
\hat{f}_{\mathbf{k}}=\sum_{\mathbf{j}}^{M} f_{\mathbf{j}} e^{-i \mathbf{k j}}
$$

and the inverse Fourier transform

$$
f_{\mathbf{j}}=\frac{1}{M} \sum_{\mathbf{k}}^{M} \hat{f}_{\mathbf{k}} e^{i \mathbf{k j}} .
$$

The distribution of $\widehat{R}_{\mathbf{k}}$ is given by

$$
\begin{aligned}
\widehat{\mathcal{P}}_{\mathrm{CN}}\left(\left\{\widehat{R}_{\mathbf{k}}\right\}\right) \propto \exp [- & \left.\frac{1}{2} \sum_{\mathbf{k}}^{M} \frac{\widehat{R}_{-\mathbf{k}} \widehat{R}_{\mathbf{k}}}{2 M \hat{\Delta}_{\mathbf{k}}}\right] \\
& =\prod_{\mathbf{k}}^{M} \exp \left[-\frac{1}{2} \frac{\widehat{R}_{-\mathbf{k}} \widehat{R}_{\mathbf{k}}}{2 M \hat{\Delta}_{\mathbf{k}}}\right] .
\end{aligned}
$$

Note that $\Delta_{\left|\mathbf{i}-\mathbf{i}^{\prime}\right|}^{-1}$ is diagonal in Fourier space: $\Delta_{\mathbf{k}}^{-1}$ $=1 / \Delta_{\mathbf{k}}$. Also note that since $R_{\mathbf{j}}$ is real, $\widehat{R}_{-\mathbf{k}}=\widehat{R}_{\mathbf{k}}^{*}$. 
Equation 42 tells us that the distributions of both $\operatorname{Re}\left(\widehat{R}_{\mathbf{k}}\right)$ and $\operatorname{Im}\left(\widehat{R}_{\mathbf{k}}\right)$ are Gaussian, each with (k dependent) variance $2 M \hat{\Delta}_{\mathbf{k}}$; hence the term "colored noise."

In Fourier space, eq 37 becomes

$$
\widehat{W}_{\mathbf{k}}^{\mathrm{T}}=\widehat{W}_{\mathbf{k}}^{m}+\hat{\Delta}_{\mathbf{k}} \widehat{F}_{\mathbf{k}}^{m}+\widehat{R}_{\mathbf{k}},
$$

where we have used the cyclic convolution theorem. Similarly, eq 39 can be expressed as

$$
\tau=\frac{\widehat{\mathcal{P}}_{\mathrm{CN}}\left(\left\{\widehat{W}_{\mathbf{k}}^{m}-\widehat{W}_{\mathbf{k}}^{\mathrm{T}}-\hat{\Delta}_{\mathbf{k}} \widehat{F}_{\mathbf{k}}^{\mathrm{T}}\right\}\right)}{\widehat{\mathcal{P}}_{\mathrm{CN}}\left(\left\{\widehat{W}_{\mathbf{k}}^{\mathrm{T}}-\widehat{W}_{\mathbf{k}}^{m}-\hat{\Delta}_{\mathbf{k}} \widehat{F}_{\mathbf{k}}^{m}\right\}\right)} e^{-\left(H^{\mathrm{T}}-H^{m}\right)} .
$$

The variance $\hat{\Delta}_{\mathbf{k}}$ is selected to be a function peaked at the wavenumber $k_{0}=2 \approx q^{*}$ roughly corresponding to $R_{g 0}$. For example,

$$
\hat{\Delta}_{\mathbf{k}}=\hat{\Delta}\left[e^{-\left(k-k_{0}\right)^{2} / 2 \sigma^{2}}+\delta\right],
$$

or

$$
\hat{\Delta}_{\mathbf{k}}=\mathcal{S}_{\mathbf{k}}=\hat{\Delta} \frac{k^{2}}{k_{0}^{4}+k^{4}}
$$

Here $k_{0}, \sigma$, and $\delta$ remain constant, while $\hat{\Delta}$ is adjusted to ensure the acceptance ratio is $\approx 0.5$. Equation 45 allows us to adjust the breadth of harmonics of the kicks by changing $\sigma$. We can introduce a uniform "white noise" background distribution by setting $\delta \neq 0$. Alternatively, eq 46, while also peaked at $k_{0}$, captures the observed low- $k$ and high- $k$ behavior of the diblock structure factor $\mathcal{S}_{\mathbf{k}} \cdot{ }^{12}$

In the next section we present results that illustrate the effectiveness of the SIS, SF, and PSP-MC algorithms at yielding defect-free states. Furthermore, we also address the possibility that the PSP-MC algorithms may represent a useful tool for examining equilibrium defect populations.

\section{RESULTS}

In the simulations reported here we applied periodic boundary conditions in both lateral directions, and we set $L_{x}=L_{y}=48 R_{g 0}, n_{x}=n_{y}=192$, $n_{s}=70, f=0.7, \chi N=17$, and $C=20$ or 100 .

For the PSP-MC algorithms, we relax $\Xi$ (using the SIS scheme) until the relative error in $H[\Xi, W]$ is below $\epsilon=0.001$. On average, approximately five SIS steps in $\Xi$ are needed for each trial MC update. In the SIS update scheme, $C$ always appears as a multiplicative factor next to $\Delta t$, and thus represents a modification to the SIS time step. In all SIS updates, the overall time step $\Delta t C$ is set to $\Delta t C=100$.

As mentioned earlier, all colored-noise SMC runs have a Fourier space noise distribution that is peaked at $k_{0}=2 \approx q^{*}$. For the Gaussian colored-noise distributions, we set $\delta=0$ (i.e., no background white noise).

For the SIS runs, we relax the system for $n_{t}=20,000$ total time steps (this ensures a well relaxed saddle point solution). For the PSP-MC runs we also run for $n_{t}=20,000$ total time steps (attempted MC moves); however, the PSPMC runs are split into two distinct sections: a 10,000 iteration equilibration run, and a 10,000 iteration production run (the value of $\Delta$ or $\hat{\Delta}$ is fixed over the production run).

For the SF runs, we apply the spectral amplitude filter at $t_{m}=5000$ with $f_{\max }=0.75$, then again at $t_{m}=6000$ with $f_{\max }=0.50$, and finally at $t_{m}=7000$ with $f_{\max }=0.25$. Before, between, and after SF applications, we relax the system toward a saddle point solution with the SIS algorithm. After the final spectral filter application, we continue to relax the system using the SIS algorithm until $t_{m}=n_{t}=20,000$.

For each scheme, we performed two independent runs, each with a different random initial condition, and at the end of the run performed a Voronoi analysis and computed the average defect density reported.

For all PSP-MC runs, we began with a random initial condition, followed by exactly five SIS steps, and then started the actual MC run. The five SIS steps are necessary because the first few initial moves away from a random initial state were observed to always increase the value of $H[\Xi, W]$, usually by such a large amount that the probability of accepting such an MC move is negligible.

The above-mentioned set of parameters is largely arbitrary; however, there were a few guidelines that we adhered to when selecting the system parameters: (1) defects vs. run time-we wanted a simulation cell large enough to exhibit significant numbers of defects (SCFT simulations on small systems often do not exhibit defects), but small enough to keep run times manageable. With this size, our runs (20,000 field iterations) lasted $\approx 20 \mathrm{~h}$ for $\mathrm{SIS} / \mathrm{SF}$ and $\approx 60 \mathrm{~h}$ for PSP-MC on a single-processor workstation. (2) We set $d x=d y$ $=0.25 R_{g 0}$ in order to ensure adequate resolution to resolve the domain interfacial regions, and we 
selected the number of contour points $\left(n_{s}=70\right)$ to be the smallest value that does not appreciably affect the solutions obtained. (3) The most detailed experimental studies of defects in thin film block copolymer systems are performed near the ODT. ${ }^{5-7}$ While we elected not to use the exact values of $f$ and $\chi N$ corresponding to the experimental studies, we selected parameters $f=0.7$ and $\chi N=17$ that not only place the system near the ODT at both $C=100$ and $C=20$, but also at a composition where small $\chi N$ variations sustain the cylindrical phase. This allowed us the opportunity to study $\chi N$ dependent effects without the requirement of changing $f$.

The procedure for obtaining defect densities involves four steps: (1) run the simulations, (2) generate the Voronoi diagrams, (3) count the number of pentagons $n_{5}$ (or heptagons $n_{7}$ ) and the total number of polygons $n_{\text {tot }}$, and (4) calculate the defect densities:

$$
\rho_{\mathrm{D}}=\frac{n_{5}}{n_{\text {tot }}}=\frac{n_{7}}{n_{\text {tot }}} .
$$

For well-relaxed systems in the hexatic or crystal phase, $n_{5}+n_{6}+n_{7}=n_{\text {tot }}$, as observed in the experiment. $^{5}$

In the present section we compare five different defect annealing schemes: SCFT implemented with SIS relaxation (SIS), spectral amplitude filtering in tandem with SIS relaxation (SF), PSP white-noise SMC, PSP colored-noise SMC with a $\sigma=1$ Gaussian noise distribution (SMC1), and PSP colored-noise SMC with $\hat{\Delta}_{\mathbf{k}}$ given by eq 46 (SMCS).

In Figure 2 we provide a representative composition pattern for a well-relaxed SIS simulation ( $\phi_{\mathrm{A}}$ in white). This system clearly exhibits a significant concentration of microdomain defects. The lowest energy mean-field density consists of a hexagonally ordered lattice of B-rich microdomains (i.e., each microdomain has 6 nearest neighbors). Here, there are various microdomains with five or seven nearest neighbors. As we shall see, the defects are more easily identified (and enumerated) with Voronoi diagrams.

In Figures 3 and 4, we provide representative Voronoi diagrams resulting from simulation runs using SIS, SF, SMC, SMC1, and SMCS. Recall that the number of sides of the polygon centered on a cylinder represents the number of nearest-neighbor cylindrical domains. Thus, hexagons (in white) represent perfect 6-coordinated microdomains, while pentagons (in light gray) and heptagons (in dark gray) represent defects (in this

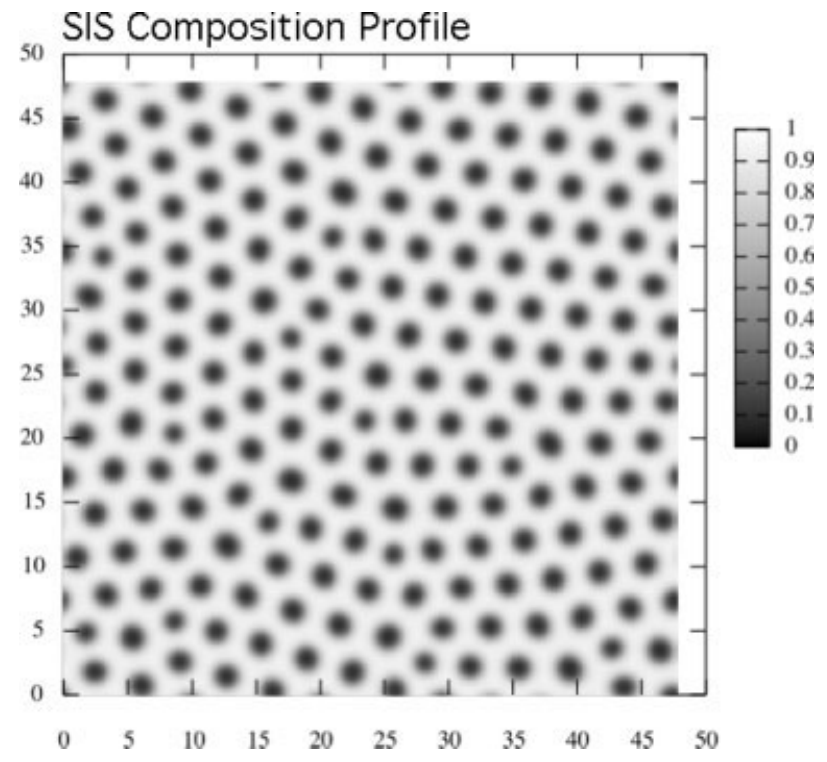

Figure 2. Composition profile, $\phi_{\mathrm{A}}$ (in white), for a wellrelaxed SCFT simulation (using SIS relaxation). Note that there are numerous microdomain defects, characterized by a breakdown of perfect sixfold coordination. The $x$ and $y$ axes are in units of $R_{g 0}$.

case disclinations). It is important to note that the relaxed SIS density has a nonzero defect density. This is a common occurrence and the primary motivation for this work.

In Figure 5 we present average pentagon density versus number of iterations $\left(t_{m}\right)$. For each of the PSP-MC relaxations, we ran with $C=100$ and $C=20$. We see from Figure 5 that the SF relaxation was the most successful of the five schemes at removing defects. In fact, for our system, SF relaxation always produced defect-free states. The second most successful algorithm was the SIS relaxation, followed by the various PSP-MC algorithms.

It is not surprising that the SF was the most successful at removing microdomain defects. The Fourier landscape of hexagonally ordered cylinders is rather simple (the first-order structure consists of six equally spaced peaks, all of which are equidistant from the $(0,0)$ mode). Consequently, the spectral amplitude filter is consistently successful at filtering out "noise" associated with microdomain defects.

It is interesting that the SIS relaxation scheme consistently outperformed the various PSP-MC algorithms in the elimination of defects. There are a few possible explanations for this observation.

First, the amplitude of the thermal fluctuations may be too large or too small. We examined the PSP-MC at two values of $C$. For $C=100$ we noticed 

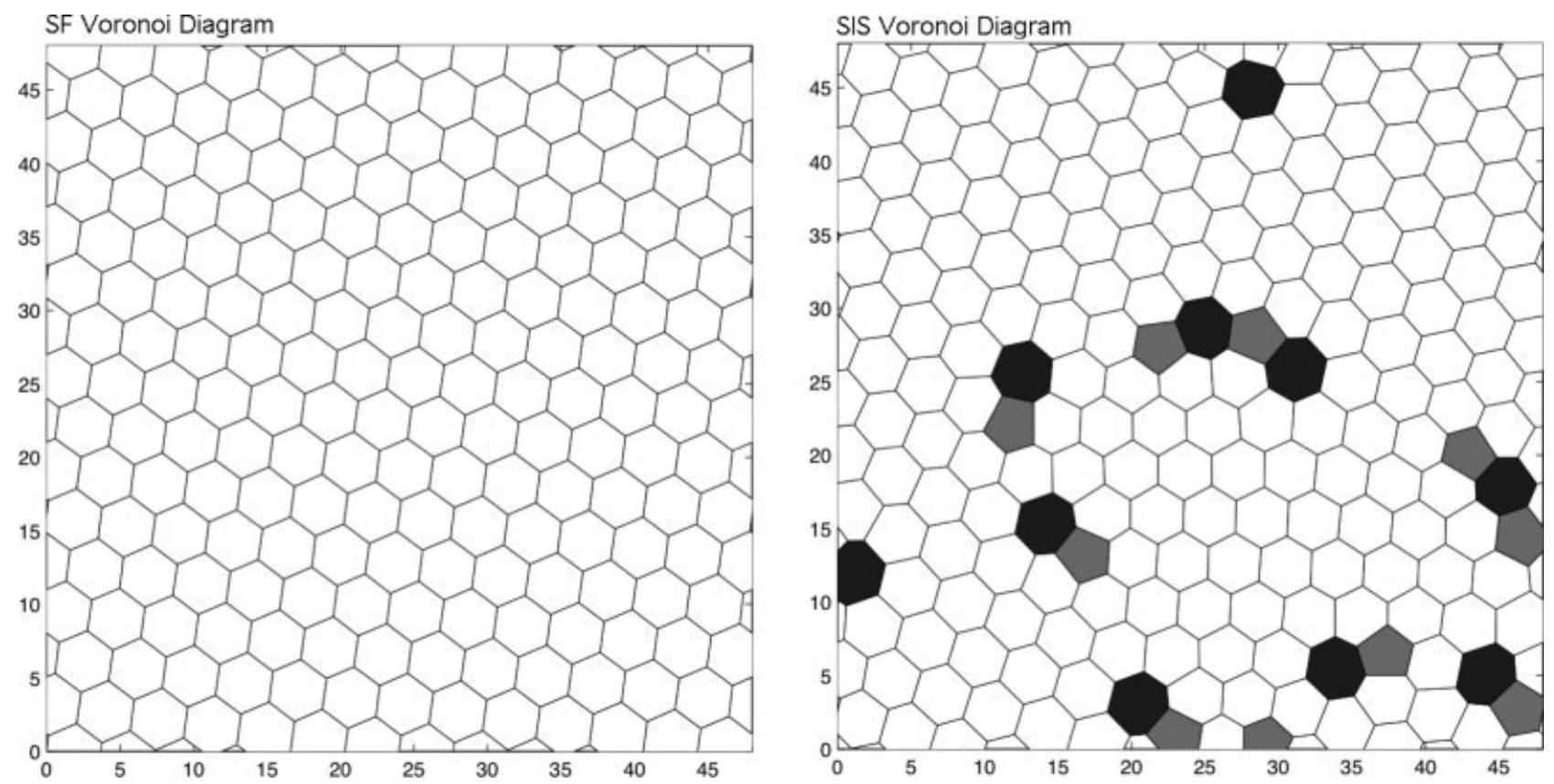

Figure 3. Representative Voronoi diagrams for SF (left) and SIS (right) relaxations at 20,000 iterations. In both figures, the $x$ and $y$ axes are in units of $R_{g 0}$.

very little microdomain movement. $C=100$ is a relatively high value (recall $C \rightarrow \infty$ gives SCFT), and thus the energy provided by the MC fluctuations may be below the energy required to "jump" out of the local minima corresponding to metastable defects. $C=20$, on the other hand, represents a rather low value where fluctuations are significant. ${ }^{26}$ In this case, the fluctuations are likely large enough that the system is continuously kicked in to and out of both local and global minima. Our observations are consistent with considerable defect motion at $C=20$. One could imagine that there exists a "sweet spot" in $C$, intermediate between $C=20$ and $C=100$, where one or all of the PSP-MC algorithms are capable of achieving states with low defect densities. More likely, however, it would be necessary to gradually ramp $C$ from a low value to a high value in a type of "simulated annealing" procedure to achieve and maintain low defect densities in such MC simulations.

Second, it is possible that we have actually realized our secondary goal of developing methods of sampling equilibrium defect populations. For each of the PSP-MC algorithms, the defect fraction appears to level off to a fixed value. This is not desirable from a defect removal standpoint; however, it is exactly what one would expect when observing equilibrium populations of defects characteristic of a $2 \mathrm{D}$ crystalline or hexatic phase.
Finally, it is also possible that the time scale for defect removal is far longer than the 20,000 iterations we have selected here. In order to partially examine this issue, we set up two of our simulations to focus intensely on moving defects. The SMC1 relaxations have a noise distribution in Fourier space that is sharply peaked at $q^{*}$. The peak is so sharp that the energies and densities yielded by the SMC1 update differ significantly from the other PSP-MC updates. It is our belief that the SMC1 updates are nonergodic, and thus unsuitable for statistical sampling of the diblock partition function. However, the SMC1 algorithm is particularly well suited for defect removal. The SMC1 algorithm was not the most successful at removing defects on the time scale examined; however, longer test runs of the SMC1 scheme (beyond 20,000 iterations) suggest that it continues to slowly remove defects (in fact, the SMC1 defect fractions approached those of the SIS relaxation). Although this may represent a partial success, the time scales are too long to be a practical method of removing defects. In addition, since the SMC1 relaxation is nonergodic, it does not represent a useful tool for examining equilibrium defect populations.

We have yet to discuss the results of the SMCS algorithm. Even though the SMCS Fourier space noise distribution is sharply peaked at $k=2 \approx q^{*}$, the method does appear to be ergodic 

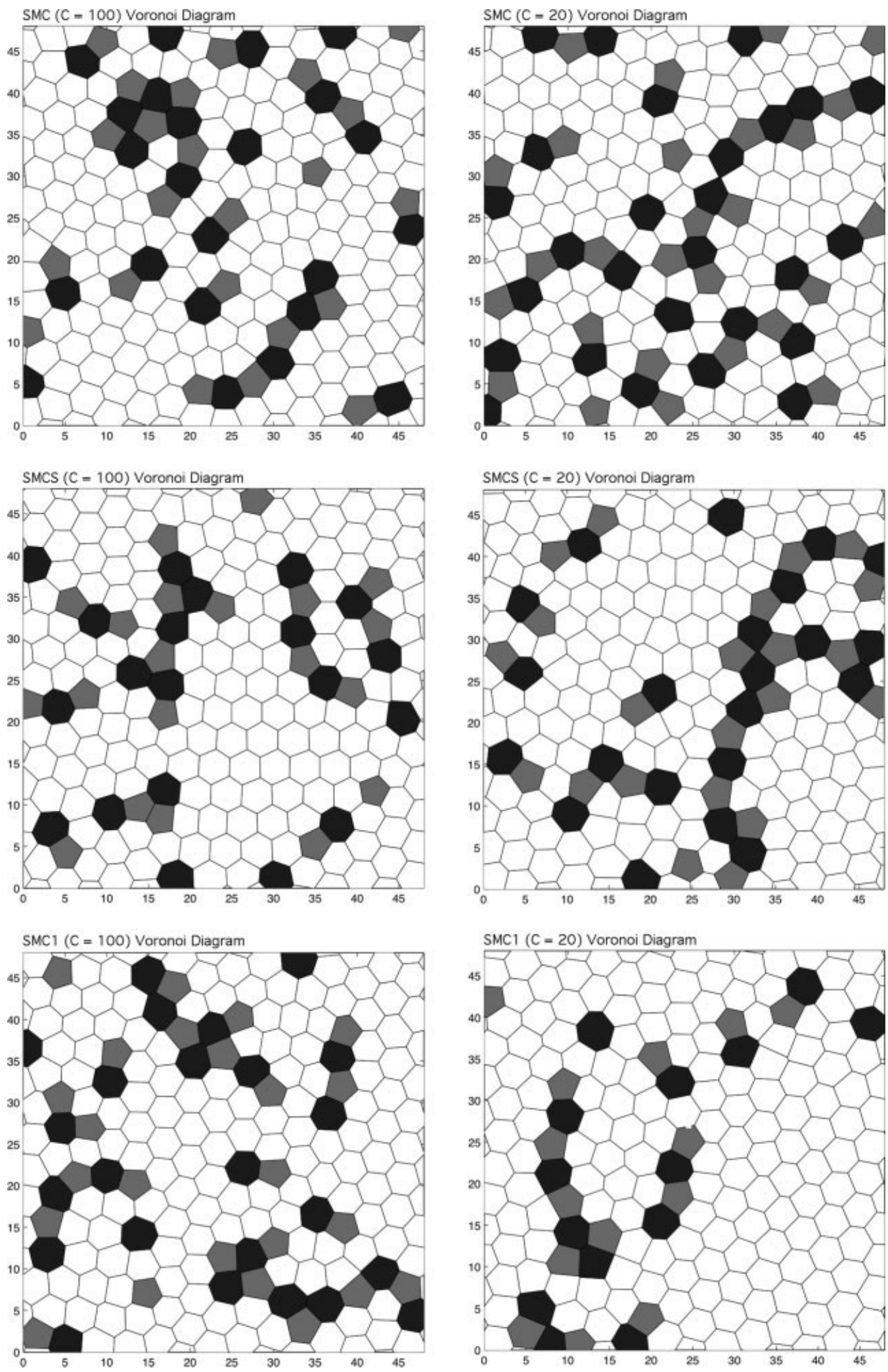

Figure 4. Representative Voronoi diagrams for SMC $(C=100)$ (top left), SMCS $(C=100)$ (middle left), SMC1 $(C=100)$ (bottom left), SMC $(C=20)$ (top right), SMCS $(C=20)$ (middle right), and SMC1 $(C=20)$ (bottom right) at 20,000 iterations. In all figures, the $x$ and $y$ axes are in units of $R_{g 0}$. 


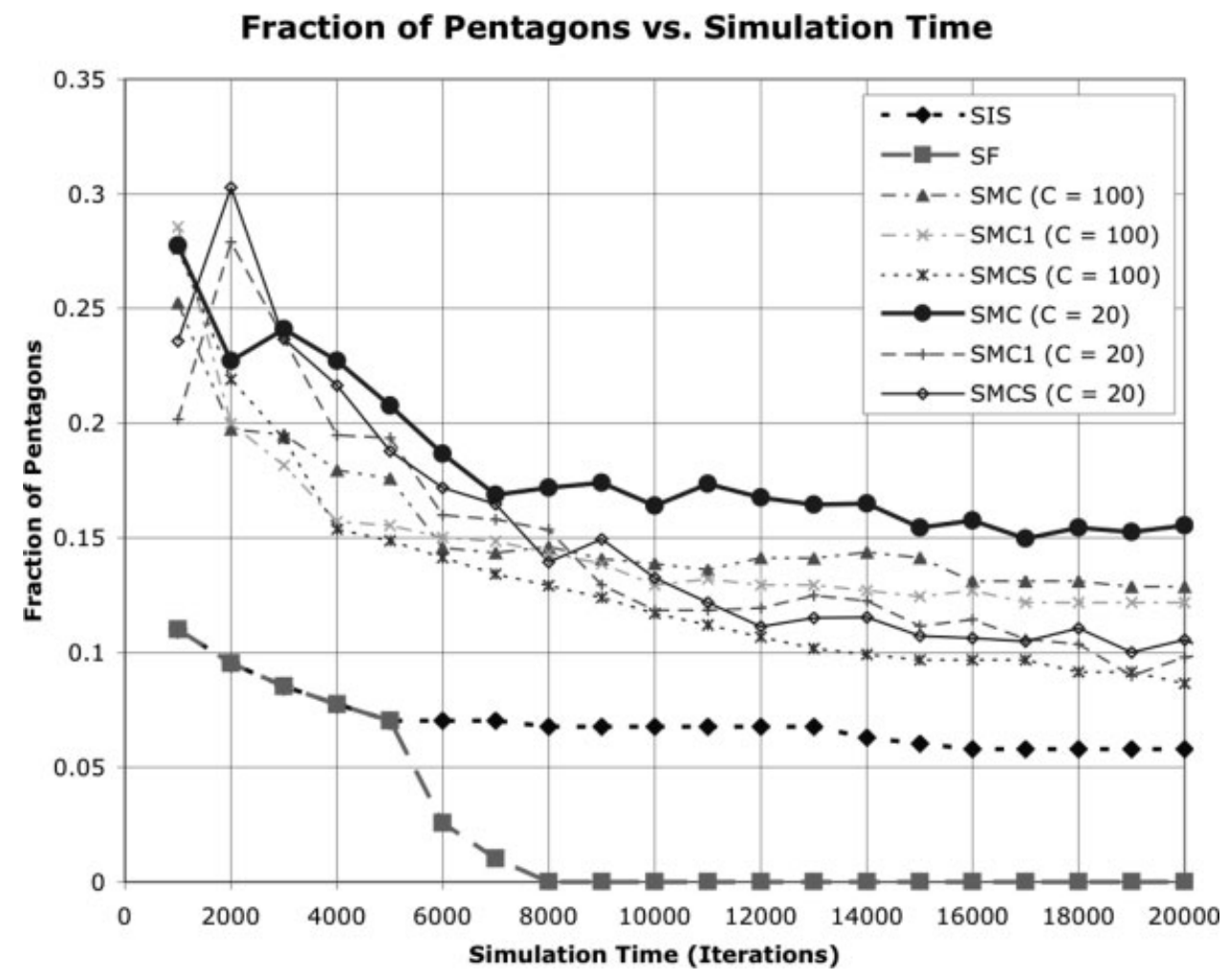

Figure 5. Fraction of pentagons vs. simulation time. The SF relaxation is the most successful at removing defects while the SMC scheme is the least successful. For the PSPMC schemes, the defect fractions appear to level off, and thus may represent equilibrium populations.

(i.e., it yielded similar density profiles and energies to other ergodic PSP-MC methods such as Metropolis MC and the white noise SMC). Of all the PSP-MC algorithms at $C=100$, the SMCS scheme was the most successful at removing defects over the time scale examined. Since this update scheme does appear to fully sample phase space, and it appears to equilibrate more rapidly than the other PSP-MC algorithms, it may represent a useful tool for examining equilibrium defect populations and possibly studying the crystal-hexatic transition in 2D block copolymer thin films. Further study will be necessary to determine the computational feasibility of such investigations.

\section{DISCUSSION AND CONCLUSIONS}

We have developed a family of field update algorithms primarily intended to systematically remove metastable defects encountered in simulations of block copolymer fluids. In our trials on $2 \mathrm{D}$ melt films of $\mathrm{AB}$ diblock copolymers with hexagonal mesophase order, the SF algorithm was the most effective at achieving defect-free, meanfield (SCFT) solutions. Our MC algorithms based on the PSP approximation (PSP-MC) incorporate thermal fluctuations, and while less effective at annealing defects, may prove useful for sampling equilibrium defect populations.

Our numerical simulations illustrate that the SF algorithm is very efficient at removing defects in the $2 \mathrm{D} \mathrm{AB}$ block copolymer systems studied. However, preliminary tests on $\mathrm{AB}$ systems in $3 \mathrm{D}$ show that the method can run into difficulty for complex block copolymer mesophases (such as gyroid), where lower amplitude Fourier peaks that are crucial for specifying symmetry can become buried in a rough landscape created by defects. It remains to be seen how useful the SF algorithm will be when applied to simulations of ABC block copolymer systems that possess large numbers of complex phases.

Experimental studies of defect populations in block copolymer thin films indicate that a single layer of spherical microdomains exhibit ordering characteristics consistent with KTHNY theory, DOI 10.1002/polb 
including equilibrium defect densities. ${ }^{5}$ The ergodic PSP-MC algorithms developed here (SMC and SMCS) appear to be useful algorithms in computational studies of equilibrium defect populations and numerical investigations of the applicability of KTHNY theory to block copolymer thin films. Of the PSP-MC variants, the SMCS method appears the most promising for such studies, as it is not only ergodic but also injects noise concentrated near wavenumbers $k=2 \approx q^{*}$ that are effective at producing defect motion. We hope to report on further investigations along these lines in the near future.

G. H. Fredrickson and A. W. Bosse derived partial support from NSF grant DMR-0312097. The work of C. J. García-Cervera was funded by NSF grant DMS0411504. A. W. Bosse received partial supported from The Frank H. and Eva B. Buck Foundation. This work made use of MRL Central Facilities supported by the MRSEC Program of the National Science Foundation under Award No. DMR05-20415. In addition, we acknowledge use of the Hewlett-Packard cluster in the CNSI Computer Facilities. We are also greatful to Gila Stein and Edward Kramer for providing the MATLAB code used to generate the Voronoi diagrams.

\section{REFERENCES AND NOTES}

1. Naito, K.; Hieda, H.; Sakurai, M.; Kamata, Y.; Asakawa, K. IEEE Trans Magn 2002, 38, 1949.

2. Park, C.; Yoon, J.; Thomas, E. L. Polymer 2003, 44, 6725.

3. Park, M.; Harrison, C.; Chaikin, P. M.; Register, R. A.; Adamson, D. H. Science 1997, 276, 1401.

4. Segalman, R. A. Mater Sci Eng R 2005, 48, 191.

5. Segalman, R. A.; Hexemer, A.; Hayward, R. C.; Kramer, E. J. Macromolecules 2003, 36, 3272.

6. Segalman, R. A.; Hexemer, A.; Kramer, E. J. Macromolecules 2003, 36, 6831 .
7. Segalman, R. A.; Yokoyama, H.; Kramer, E. J. Adv Mater 2001, 13, 1152.

8. Binder, K. Ed. Monte Carlo and Molecular Dynamics Simulations in Polymer Science; Oxford University Press: New York, 1995.

9. Fredrickson, G. H. The Equilibrium Theory of Inhomogenous Polymers; Clarendon Press: Oxford, 2006.

10. Matsen, M. W.; Schick, M. Phys Rev Lett 1994, 72, 2660.

11. Bates, F. S.; Fredrickson, G. H. Ann Rev Phys Chem 1990, 42, 525.

12. Leibler, L. Macromolecules 1980, 13, 1602.

13. Rasmussen, K. O.; Kalosakas, G. J Polym Sci B: Polym Phys 2002, 40, 1777.

14. Sides, S. W.; Fredrickson, G. H. Polymer 2003, 44, 5859.

15. Ceniceros, H. D.; Fredrickson, G. H. Multiscale Model Simul 2004, 2, 452.

16. Alexander-Katz, A.; Moreira, A. G.; Fredrickson, G. H. J Chem Phys 2003, 118, 9030.

17. Fredrickson, G. H.; Ganesan, V.; Drolet, F. Macromolecules 2002, 35, 16.

18. Lennon, E.; Katsov, K.; Ceniceros, H. D.; GarcíaCervera, C. J.; Fredrickson, G. H. Unpublished work.

19. Knoll, A.; Lyakhova, K. S.; Horvat, A.; Krausch, G.; Sevink, G. J. A.; Zvelindovsky, A. V.; Magerle, R. Nature Mater 2004, 3, 886.

20. Sakurai, J. J. Modern Quantum Mechanics, Revised ed. Addison Wesley: Reading, MA, 1994.

21. Frigo, M.; Johnson, S. G. Proc IEEE 2005, 93, 216.

22. Sides, S. W.; Fredrickson, G. H. Unpublished work.

23. Düchs, D.; Ganesan, V.; Fredrickson, G. H.; Schmid, F. Macromolecules 2003, 36, 9237.

24. Reister, E.; Müller, M.; Binder, K. Phys Rev E 2001, 64, No. 041804.

25. Rossky, P. J.; Doll, J. D.; Friedman, H. L. Chem Phys 1978, 69, 4628.

26. Ganesan, V.; Fredrickson, G. H. Europhys Lett 2001, 55,814 . 